\title{
Inaccuracy of lung adenocarcinoma subtyping using preoperative biopsy specimens
}

\author{
Kuo-Yang Huang, MD, ${ }^{\mathrm{a}}$ Pin-Zuo Ko, MD, ${ }^{\mathrm{b}}$ Chih-Wei Yao, MD, ${ }^{\mathrm{c}}$ Cheng-Nan Hsu, MD, ${ }^{\mathrm{d}}$ \\ Hsin-Yuan Fang, MD, ${ }^{\text {ef }}$ Chih-Yeh Tu, MD, ${ }^{\text {f,g }}$ and Hung-Jen Chen, MD $^{\mathrm{g}, \mathrm{h}}$
}

\begin{abstract}
Background: The prognostic significance of the new classification of lung adenocarcinoma proposed in the 2015 World Health Organization guideline has been validated. This study aimed to compare the preoperative classification of the adenocarcinoma subtype based on computed tomography-guided 18-gauge core needle biopsy (CTNB) or radial probe endobronchial ultrasound (REBUS) specimens, with the postoperative classification based on the resected specimens.
\end{abstract}

Methods: We retrospectively analyzed a consecutive series of 128 patients (60 CTNB and 68 R-EBUS) who underwent surgery for preoperatively confirmed lung adenocarcinoma between 2010 and 2014. Comprehensive histological subtyping was performed according to the 2015 World Health Organization classification system. Diagnostic concordance of subtypes between small biopsy and resection specimens was assessed.

Results: Concordant subtyping of adenocarcinomas between the predominant pattern on resections and biopsy sections was observed in $58.6 \%$ of cases $(75$ of $128 ; 95 \%$ confidence interval [CI], 49.9\%-66.8\%). Preoperative subtyping was accurate in only $30 \%$ of samples (3 of 10) with a predominance of solid patterns. None of the 5 micropapillary predominant cases was detected by CTNB or R-EBUS. For the concordance of the presence or absence of micropapillary/solid component, the sensitivity was as low as $16.5 \%(95 \% \mathrm{CI}, 9.1 \%-26.5 \%)$. The detection rate by CTNB/R-EBUS increased with the increase in the percentage of micropapillary/solid component; however, even in the $\geq 40 \%$ micropapillary/solid group, only $24 \%$ of cases were detected by CTNB/R-EBUS.

Conclusions: The accuracy of the estimation of adenocarcinoma histological subtype based on preoperative biopsy sections was unsatisfactory. (J Thorac Cardiovasc Surg 2017;154:332-9)

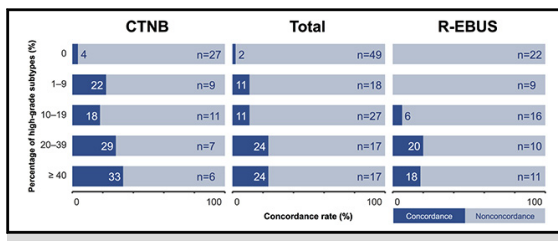

Detection of high-grade adenocarcinomas by preoperative biopsy section.

\section{Central Message}

The accuracy of estimation of adenocarcinoma histological subtype based on preoperative biopsy sections was unsatisfactory.

\section{Perspective}

The identification of the presence or absence of micropapillary/solid components of an earlystage lung adenocarcinoma preoperatively is a crucial but unresolved issue. We show that the accuracy of estimation based on preoperative biopsy sections was unsatisfactory. Surgeons should not rely solely on preoperative biopsy findings when making decisions regarding the choice of limited resection or lobectomy.

See Editorial Commentary page 340 .

\footnotetext{
From the ${ }^{\text {a Division }}$ of Chest Medicine, Department of Internal Medicine, Yuanlin Christian Hospital, Changhua; Departments of ${ }^{\mathrm{b}}$ Pathology and ${ }^{\mathrm{d}}$ Radiology, and ${ }^{\mathrm{e}}$ Division of Thoracic Surgery, Department of Surgery, ${ }^{\mathrm{f}}$ School of Medicine, College of Medicine, and ${ }^{\mathrm{g} D i v i s i o n}$ of Pulmonary and Critical Care, Department of Internal Medicine, China Medical University Hospital; ${ }^{\mathrm{c} D i v i s i o n}$ of Chest Medicine, Department of Internal Medicine, Everan Hospital; and ${ }^{\mathrm{h}}$ Department of Respiratory Therapy, China Medical University, Taichung, Taiwan.

P-Z.K. and C-W.Y. contributed equally to this work.

Received for publication Aug 21, 2016; revisions received Jan 19, 2017; accepted for publication Feb 19, 2017; available ahead of print March 31, 2017.

Address for reprints: Hung-Jen Chen, MD, Division of Pulmonary and Critical Care, Department of Internal Medicine, China Medical University Hospital, No. 2, Yude Rd, North District, Taichung City 40402, Taiwan (E-mail: redman0127@gmail. com).

$0022-5223 / \$ 36.00$

Copyright (c) 2017 by The American Association for Thoracic Surgery

http://dx.doi.org/10.1016/j.jtcvs.2017.02.059
}

Adenocarcinoma is the most common subtype of lung cancer, which is the leading cause of cancer-related deaths worldwide. ${ }^{1}$ However, adenocarcinoma is a heterogeneous disease with variant morphological features. To predict clinical prognosis, the International Association for the Study of Lung Cancer, the American Thoracic Society, the

Scanning this QR code will take you to a supplemental video, figure, and table for the article.

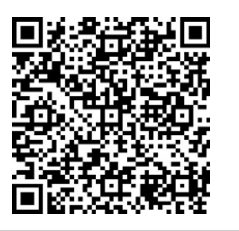




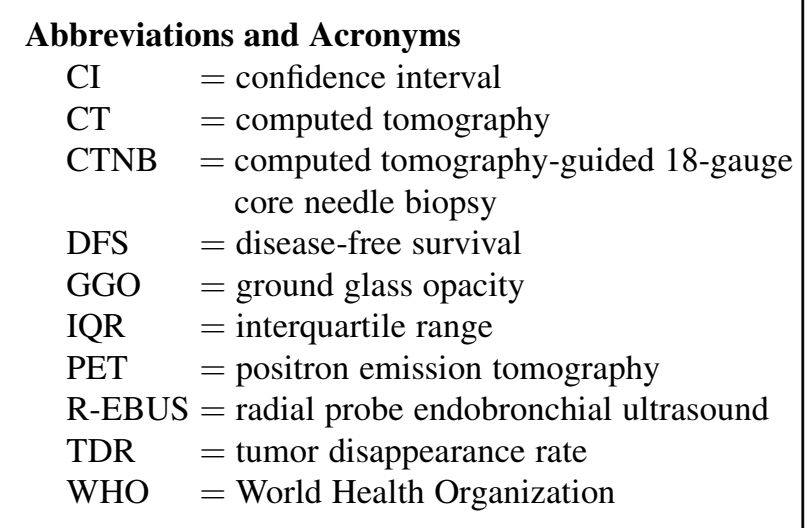

European Respiratory Society, and the 2015 World Health Organization (WHO) guidelines classify adenocarcinomas based on the molecular characteristics and predominant architectural subtypes as lepidic, acinar, papillary, micropapillary, or solid. ${ }^{2,3}$ These classification systems recommend comprehensive histological subtyping based on pattern recognition and a semiquantitative assessment of each pattern in 5\% increments for evaluating the single predominant pattern in invasive adenocarcinomas. ${ }^{2,3}$

The predominant architectural subtype of adenocarcinomas is correlated with clinical prognosis. Nakamura et $\mathrm{al}^{4}$ reported that standardized fluorodeoxyglucose uptake values for positron emission tomography (PET)/computed tomography (CT) were higher in adenocarcinomas with predominant micropapillary and solid patterns. In early-stage lung adenocarcinomas, following complete resection, predominant micropapillary and solid subtypes have been associated with poorer disease-free survival (DFS), whereas a predominant lepidic subtype was associated with favorable outcomes. ${ }^{5,6}$

Even a small proportion of micropapillary or solid component has a significant negative impact on prognosis. ${ }^{7-9}$ In clinically N2-negative lung adenocarcinoma, the presence of micropapillary or solid component was associated with pathological N2 disease. ${ }^{10,11}$ Furthermore, the percentage of micropapillary or solid component increased with the increasing likelihood of pathological N2 disease. ${ }^{10}$ Lymph node metastasis is one of the most important factors in determining the treatment plan for a resectable lung adenocarcinoma. Patients with pathological N2 stage IIIA lung adenocarcinoma who received neoadjuvant therapy before surgery showed better outcomes compared with those who underwent primary surgical resection followed by adjuvant therapy. ${ }^{12}$ Therefore, precise preoperative staging integrated with PET/CT, endobronchial ultrasoundguided transbronchial needle aspiration, or endoscopic ultrasound-guided fine-needle aspiration ${ }^{13}$ may be performed to increase the reliability of preoperative pathological N2 detection in patients with micropapillary or solid component lung adenocarcinoma. In contrast, predominant early-stage lepidic adenocarcinomas demonstrated better prognosis. ${ }^{5,6}$ Although lobectomy is a standard treatment for resectable lung adenocarcinomas, sublobar resection may be an option for elderly patients and those with a $\leq 2$-cm tumor and predominant lepidic subtype. ${ }^{14}$

Therefore, if a preoperative small biopsy specimen were capable of replacing an operative large specimen for subtyping lung adenocarcinoma, this could have a significant impact for surgeons. For example, if the preoperative small biopsy specimen by CT-guided 18-gauge core needle biopsy (CTNB) or radial probe endobronchial ultrasound (REBUS) could be used to accurately subtype lung adenocarcinoma, then sublobar resection might not be an option for patients with a small peripheral lung adenocarcinoma containing micropapillary/solid components. For elderly patients with lung adenocarcinoma presenting with groundglass opacity (GGO), surgeons could change their decisions regarding lobectomy in cases where a predominantly acinar subtype was proven by perioperative CTNB. Therefore, the identification of the predominant lepidic subtype and presence or absence of micropapillary/solid component of a lung adenocarcinoma, either preoperatively or intraoperatively, is a crucial but unresolved issue for surgeons.

Bittar et a ${ }^{15}$ and Yeh et al ${ }^{16}$ have reported that it is difficult to predict a primary adenocarcinoma subtype in a frozen sample that is intraoperatively collected because of poor quality and sampling artifacts. Accurate subtyping of non-smallcell lung cancer into squamous cell lung cancer or adenocarcinoma can be achieved by CTNB or R-EBUS. ${ }^{17,18}$ The accuracy of small biopsy subtyping of adenocarcinoma has not yet been completely validated, however. ${ }^{19}$

Therefore, in this study, we aimed to compare the preoperative biopsy specimens by CTNB or R-EBUS with the postoperative classification of resected specimens with 3 objectives. First, we investigated the concordance of predominant subtype classification of lung adenocarcinoma. Second, we investigated the concordance of the presence or absence of micropapillary/solid component. Third, we investigated the lung cancer stage and radiologic parameters that could predict the concordance of the presence or absence of micropapillary/solid components.

\section{MATERIALS AND METHODS}

\section{Study Participants}

The medical records of 580 patients who underwent surgery for lung cancer between January 2010 and December 2014 at the China Medical University Hospital in Taichung, Taiwan were retrieved from a prospective registry database and were reviewed retrospectively. The decision to obtain preoperative specimens was routinely made at the discretion of the individual clinician. Generally, R-EBUS was performed as a part of the diagnostic workup of near-central pulmonary nodules, whereas CTNB was performed for peripheral pulmonary nodules. Before each invasive biopsy procedure, the benefits and risks were explained and signed informed consent was obtained from each patient. The resected samples of primary lung cancer were 
collected intraoperatively from 163 patients, and diagnosis via preoperative biopsy histology was performed within 1 month before surgery. Of these 163 patients, 35 (29 with nonadenocarcinoma lung cancer, 2 who had received chemotherapy and/or radiotherapy, and 4 who underwent preoperative ultrasound-guided biopsy) were excluded; thus, the study population comprised 128 samples, including 60 obtained by CTNB and 68 obtained by R-EBUS (Figure E1). All R-EBUS procedures were performed or supervised by the same pulmonologist (C.-Y.-T.), and all CTNB procedures were performed by the same radiologist (C.-N.-H.), to ensure consistent quality of the procedure.

As a part of postoperative routine care, patients were followed up at least every 3 months to evaluate cancer recurrence and survival. Chest $\mathrm{CT}$ was performed every 3 months until disease progression, and long-term follow-up was performed until September 30, 2016.

The study protocol was approved by the Investigational Review Board of China Medical University Hospital (approval no. CMUH103-REC1124).

\section{Review of Hematoxylin and Eosin-Stained Preoperative and Permanent Sections}

Preoperative biopsy specimens were compared with the surgically resected specimens to evaluate the accuracy of subtype classification. Comprehensive histological subtyping was interpreted by a pathologist (P.-Z.-K.) who was blinded to the clinical data. If P.-Z.-K. had queries, he consulted with another pathologist (G.-C.-T.). Consensus on the final interpretation was reached by discussion between the 2 pathologists.

Histological subtyping was done according to the 2015 WHO lung adenocarcinoma classification system. ${ }^{2}$ The predominant histological subtype was selected as the one with the highest percentage. Micropapillary and solid patterns were defined as high-grade adenocarcinomas. ${ }^{5-9}$

\section{Review of CT Parameters}

CT scans and R-EBUS/CTNB performed within 1 month before surgery were identified. CT images were evaluated retrospectively by a single radiologist (C.-N.-H.) with more than 10 years of experience in thoracic CT imaging, who was blinded to the histopathological and clinical findings. The radiologic parameters were assessed using thin-section CT with a $1.25-\mathrm{mm}$ slice thickness and $512 \times 512$-pixel resolution. Based on a previously published study, ${ }^{20}$ the radiologic characteristics, including margin (welldefined or ill-defined), border (lobulated or nonlobulated), spiculation, tumor solidity (solid, part-solid, or pure GGO), consolidation area (largest solid diameter), and tumor disappearance rate (TDR), were reviewed, and the same definitions of consolidation, TDR, and GGO were used.

\section{Three Major Steps of the Study}

The concordance of histological subtype in the preoperative lung adenocarcinoma biopsy specimens and available permanent specimens was assessed. The first step in the study was to analyze the preoperative biopsy sections for predicting the predominant histological subtypes. The second step was to analyze the preoperative biopsy sections for predicting the presence or absence of high-grade adenocarcinoma. The third step was to evaluate the lung cancer stage and CT imaging factors that influence the ability to accurately identify high-grade adenocarcinomas via preoperative biopsy sections.

\section{Statistical Analyses}

Continuous variables are expressed as median and interquartile range (IQR; 25th and 75th percentiles), and categorical variables are expressed as number (percentage). Dichotomous variables were compared using Fisher's exact test or the $\chi^{2}$ test, as appropriate. Sensitivity, specificity, positive predictive value, and negative predictive value were calculated according to the standard definitions for subtype classification. In brief, sensitivity refers to the percentage of lung adenocarcinoma patients who test positive for a certain subtype and actually have that subtype, and specificity is the percentage of lung adenocarcinoma patients without a certain subtype who test negative for that subtype. In this nonbinary variable study, when we tested subtype A, we considered non-A subtype as a separate group.

In the subgroup DFS analysis, we examined the impact of high-grade adenocarcinoma on DFS in stage I or II patients. The survival probability was estimated using the Kaplan-Meier method, and differences among the subgroups were estimated using the log-rank test. The running logrank test was used to determine the cutoff threshold percentage of highgrade adenocarcinoma in the permanent pathological specimen. DFS was calculated from the date of the operation until the date of cancer recurrence.

A 2 -sided $P$ value $<.05$ was considered statistically significant. Statistical analyses were performed using mainly MedCalc for Windows version 15.6.1 (MedCalc Software, Ostend, Belgium). The survival analysis was performed with Stata 12.1 (StataCorp, College Station, Tex).

\section{RESULTS}

Our cohort comprised 128 patients, of whom 79 were female $(62 \%)$, with a mean age of 61.5 years (range, 3485 years). The median duration of follow-up was 28.6 months (IQR, 18.9-38.5 months). Of these, 77 patients $(60.2 \%)$ had stage I disease, $20(15.6 \%)$ had stage II disease, and $31(24.2 \%)$ had stage III or IV disease. Fourteen patients underwent wedge resection, 1 patient underwent segmentectomy, 1 patient underwent pneumonectomy, and 112 patients underwent lobectomy. Table 1 summarizes the histological patterns of the 128 surgical resection specimens. The most predominant pattern was acinar $(\mathrm{n}=70$; $54.7 \%)$, followed by papillary $(\mathrm{n}=38 ; 29.7 \%)$, solid $(\mathrm{n}=10 ; 7.8 \%)$, lepidic $(\mathrm{n}=5 ; 3.9 \%)$, and micropapillary $(\mathrm{n}=5 ; 3.9 \%)$. No patient had a single-pattern adenocarcinoma. The most common patterns of mixed adenocarcinoma were acinar $(\mathrm{n}=126 ; 98.4 \%)$ and papillary $(\mathrm{n}=98 ; 76.6 \%)$, followed by lepidic $(\mathrm{n}=71 ; 55.5 \%)$, micropapillary $(\mathrm{n}=51 ; 39.8 \%)$, and solid $(\mathrm{n}=41 ; 32.0 \%)$. High-grade adenocarcinoma, either micropapilliary or solid component, was present in 79 patients $(61.7 \%)$. The median percentage of high-grade adenocarcinoma involvement was $15 \%$ (IQR, 10\%-35\%).

\section{Survival Analyses in Association With High-Grade Adenocarcinoma Subtypes}

At the time of analysis, in stage I and II patients, local recurrence and distant metastasis had occurred in 11 and 15 patients, respectively. We performed a survival analysis according to the presence or absence of high-grade adenocarcinoma. Stage I patients without micropapillary/solid adenocarcinoma had a favorable prognosis, whereas those with micropapillary/solid adenocarcinoma had a poor prognosis. The impact of high-grade adenocarcinoma on DFS was not significant in stage II patients (Table E1).

We also observed that the presence of higher percentages of high-grade adenocarcinoma can associate with DFS. For stage I patients, statistically significant impact of highgrade adenocarcinoma on DFS reached a plateau at $40 \%$ 
TABLE 1. Clinicopathological characteristics of the study patients

\begin{tabular}{|c|c|c|}
\hline Variable & Median (IQR) & n $(\%)$ \\
\hline All patients & & $128(100)$ \\
\hline Age (y) & $62.0(53.3-71.0)$ & \\
\hline \multicolumn{3}{|l|}{ Sex } \\
\hline Female & & $79(61.7)$ \\
\hline Male & & $49(38.3)$ \\
\hline \multicolumn{3}{|l|}{ Stage } \\
\hline IA & & $37(28.9)$ \\
\hline IB & & $40(31.3)$ \\
\hline IIA & & $14(10.9)$ \\
\hline IIB & & $6(4.7)$ \\
\hline III & & $20(15.6)$ \\
\hline IV & & $11(8.6)$ \\
\hline Maximum tumor size (mm) & $28.8(21.4-36.2)$ & \\
\hline Consolidation tumor size (mm) & $27.5(20.5-36.2)$ & \\
\hline \multicolumn{3}{|c|}{ Predominance of histological patterns } \\
\hline Lepidic & & $5(3.9)$ \\
\hline Acinar & & $70(54.7)$ \\
\hline Papillary & & $38(29.7)$ \\
\hline Micropapillary & & $5(3.9)$ \\
\hline Solid & & $10(7.8)$ \\
\hline \multicolumn{3}{|c|}{ Presence of histological patterns ( $\%)$} \\
\hline Lepidic & $15(5-25)$ & $71(55.5)$ \\
\hline Acinar & $50(25-70)$ & $126(98.4)$ \\
\hline Papillary & $30(15-50)$ & $98(76.6)$ \\
\hline Micropapillary & $10(5-20)$ & $51(39.8)$ \\
\hline Solid & $15(5-45)$ & $41(32.0)$ \\
\hline \multicolumn{3}{|l|}{ Operation } \\
\hline Segmentectomy & & $1(0.8)$ \\
\hline Wedge resection & & $14(10.9)$ \\
\hline Lobectomy & & $112(87.5)$ \\
\hline Pneumonectomy & & $1(0.8)$ \\
\hline
\end{tabular}

(Table E1). The 5-year DFS for stage I patients with $<40 \%$ high-grade adenocarcinoma was $71 \%$, whereas it was $33 \%$ for those with $\geq 40 \%$ (Figure 1 ).

\section{Concordance of Adenocarcinoma Subtype Between Surgical and Preoperative Biopsy Specimens}

Concordant subtyping of adenocarcinomas between the predominant patterns on resection and CTNB/R-EBUS biopsy sections was observed in $58.6 \%$ of cases ( 75 of 128 ; $95 \%$ confidence interval $[\mathrm{CI}], 49.9 \%-66.8 \%$ ), including $61.7 \%$ (37 of $60 ; 95 \%$ CI, $49.0 \%-72.9 \%$ ) of CTNB and $55.9 \%$ (38 of $68 ; 95 \%$ CI, $44.1 \%-67.1 \%$ ) of R-EBUS (Figure 2). Acinar-predominant adenocarcinomas showed higher sensitivity $(85.7 \%)$ but lower specificity $(31.0 \%)$. The other predominant patterns showed lower sensitivity but higher specificity (lepidic pattern: sensitivity, 40.0\%, specificity, $96.8 \%$; papillary pattern: sensitivity, $26.3 \%$, specificity, $94.4 \%$; micropapillary pattern: sensitivity, $0 \%$, specificity, $100.0 \%$; solid pattern: sensitivity, $30.0 \%$, specificity, 96.6\%) (Table 2).

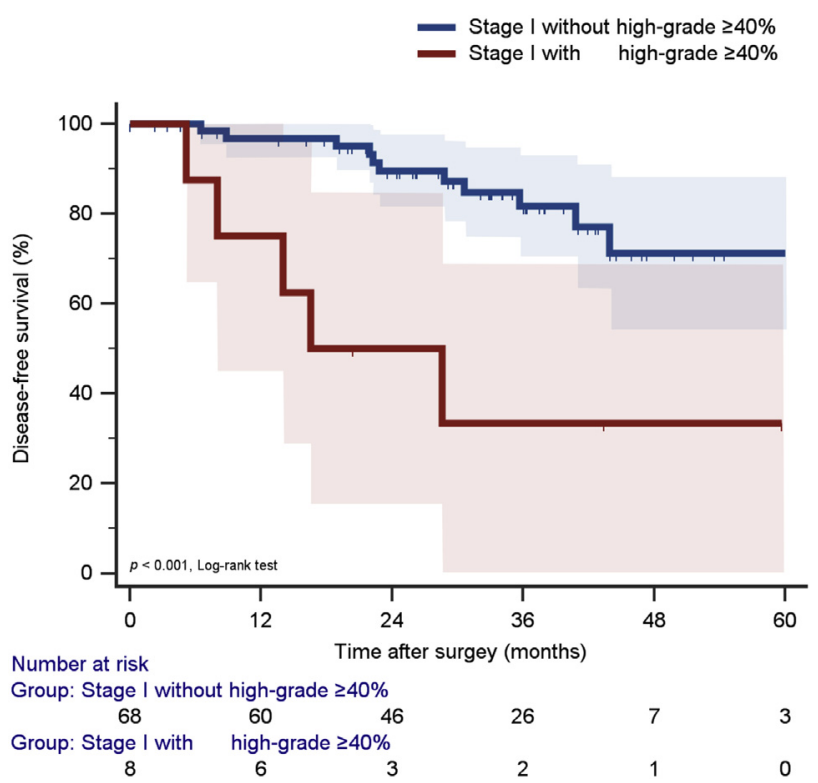

FIGURE 1. Kaplan-Meier survival curves. Disease-free in stage I according to the presence or absence of $\geq 40 \%$ micropapillary/solid component.

Analysis of the preoperative biopsy sections for predicting the presence or absence of high-grade adenocarcinoma revealed concordant subtyping of adenocarcinomas on resection and CTNB/R-EBUS biopsy sections in $47.7 \%$ of cases ( 61 of $128 ; 95 \% \mathrm{CI}, 39.2 \%-56.3 \%$ ), including $56.7 \%$ (34 of $60 ; 95 \%$ CI, $44.1 \%-68.4 \%$ ) of CTNB and $39.7 \%$ ( 27 of $68 ; 95 \%$ CI, $28.9 \%-51.6 \%$ ) of R-EBUS. The specificity was high $(98.0 \%$; 95\% CI, 89.2\%$99.9 \%)$, but the sensitivity was low $(16.5 \% ; 95 \% \mathrm{CI}$, 9.1\%-26.5\%) (Table 3). There was a more consistent agreement and higher sensitivity with CTNB sections than with R-EBUS sections for the detection of high-grade adenocarcinoma $(24.2 \%$ vs $10.9 \%)$.

The reclassification of the 128 adenocarcinoma specimens resulted in $49(38.3 \%)$ absent micropapillary/solid components, $18(14.1 \%)$ with $1 \%$ to $9 \%$ micropapillary/ solid components, $27(21.1 \%)$ with $10 \%$ to $19 \%$ micropapillary/solid components, $17(13.3 \%)$ with $20 \%$ to $39 \%$ micropapillary/solid components, and $17(13.3 \%)$ with $\geq 40 \%$ micropapillary/solid components. The detection rate by CTNB/R-EBUS increased with the increase in the percentage of micropapillary/solid components. For subgroup observation, CTNB was better than R-EBUS; however, even in the $\geq 40 \%$ micropapillary/solid group, the detection rate by CTNB was only $33 \%$ (Figure 3 and Video 1).

Lung Cancer Stage and Radiologic Parameters for Predicting the Accuracy of Detecting High-Grade Adenocarcinoma by Preoperative Biopsy Specimens

Regarding the radiologic evaluation of attenuation features, most adenocarcinomas were solid $(80.9 \%)$, whereas 

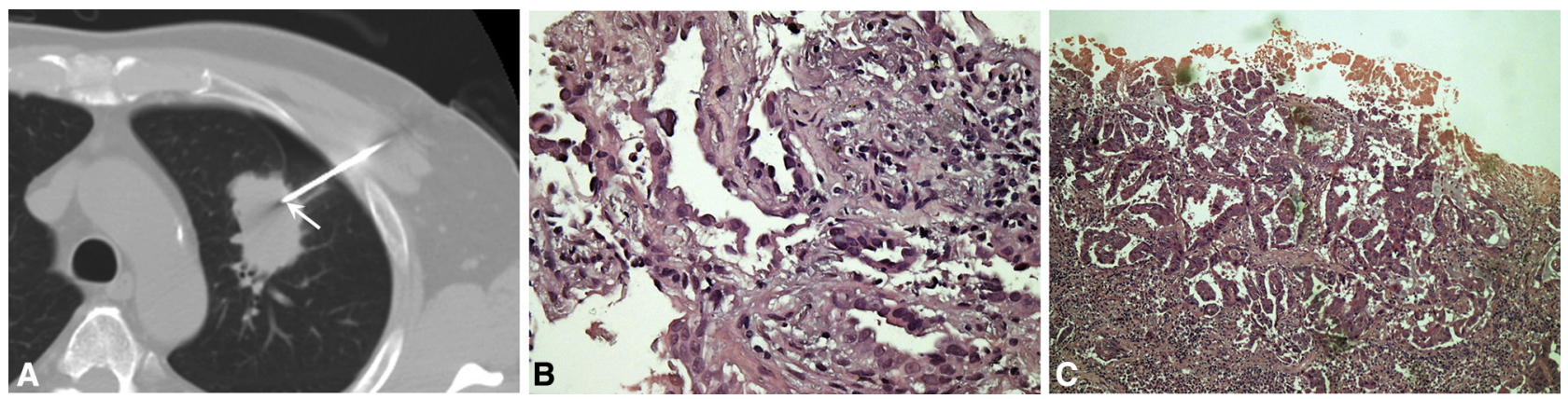

FIGURE 2. Representative case of computed tomography (CT)-guided 18-gauge core needle biopsy of lung adenocarcinoma. (A) C-arm CT scan obtained after biopsy needle insertion ensures that the needle tip (arrow) is located within the ill-defined mass in the left upper lobe. (B) This core biopsy specimen shows an adenocarcinoma comprising tumor cells presenting with acinar pattern alone (hematoxylin and eosin stain; original magnification $400 \times$ ). $(\mathrm{C}) \mathrm{A}$ permanent resection specimen revealing tumor cells arranged predominantly in a micropapillary pattern (hematoxylin and eosin stain; original magnification $100 \times)$.

only $1.6 \%$ adenocarcinomas exhibited a GGO configuration. The remaining $17.4 \%$ cases were categorized as semisolid. We classified cases according to the success or failure in predicting the presence or absence of high-grade adenocarcinoma by preoperative biopsy specimens. TDR was the sole parameter to differ between the 2 subgroups $(P=.02)$. Stage (IA vs IB-IV) and tumor size had no influence on concordance $(P=.41$ and .26 , respectively) (Table 4$)$.

\section{DISCUSSION}

To the best of our knowledge, this is the first study to address the correlation between preoperative biopsy and permanent resection sections for the concordance of highgrade adenocarcinoma detection. The results show that the accuracy of estimation of adenocarcinoma histological subtype based on preoperative biopsy sections was unsatisfactory, with a concordance of the predominant pattern of $58.6 \%$. For high-grade adenocarcinoma detection, preoperative biopsy sections had low sensitivity $(16.5 \%)$. The TDR parameter in thin-section CT showed a slight correlation with the concordance result of high-grade adenocarcinoma detection.

The standard surgical procedure for early-stage lung cancer is lobectomy. For stage I lung adenocarcinomas, limited resection has the advantage of preserving pulmonary function and may be performed in patients with a contraindication for lobar resection ${ }^{21}$ or with indolent and nodenegative lung adenocarcinoma. ${ }^{14}$ In contrast, the local recurrence rate and prevalence of extrathoracic metastases were higher in predominantly micropapillary and solid patterns of early-stage lung adenocarcinomas undergoing resection. ${ }^{7,8,22}$ Furthermore, Nitadori et $\mathrm{al}^{23}$ demonstrated that the presence of a histological micropapillary pattern in lung adenocarcinomas treated with limited resection leads to higher rates of local recurrence compared with those treated by lobectomy. This finding suggests that limited resection is not appropriate for high-grade adenocarcinomas. Thus, these findings highlight the importance of anticipating patient prognosis by the surgeon and matching it to an appropriate type of resection. ${ }^{24}$

Micropapillary/solid components, which occur in $<5 \%$ of all adenocarcinomas, have a significant negative impact on prognosis. ${ }^{7-9}$ In our study, the prevalence of micropapillary and solid components in resectable lung adenocarcinomas was $39.8 \%$ and $32.0 \%$, respectively. Similar results have been reported in previous studies. ${ }^{8,25}$ In our study, we reconfirmed the correlation between the presence of micropapillary or solid components and consistent poor DFS in patients with stage I lung adenocarcinoma. Although the optimal cutoff value of the

TABLE 2. Concordance among subtypes between small biopsy and resection specimens

\begin{tabular}{lcccccc}
\hline & \multicolumn{5}{c}{ CTNB and R-EBUS specimens, $\mathbf{n}$} & \\
\cline { 2 - 5 } & Lepidic & Acinar & Papillary & Micropapillary & Solid & Total, $\mathbf{n}(\%)$ \\
\hline Surgical specimens, $n$ & & & & & \\
$\quad$ Lepidic & 2 & 3 & 0 & 0 & 0 & $5(3.9)$ \\
Acinar & 4 & 60 & 3 & 0 & 3 & $70(54.7)$ \\
Papillary & 0 & 28 & 10 & 0 & 0 & $38(29.7)$ \\
Micropapillary & 0 & 3 & 1 & 0 & $5(3.9)$ \\
Solid & 0 & 6 & 1 & 0 & 1 \\
Total, $\mathrm{n}(\%)$ & $6(4.7)$ & $100(78.1)$ & $15(11.7)$ & $0(0)$ & $7.8)$ \\
\hline
\end{tabular}

$C T N B$, Computed tomography 18-gauge core needle biopsy; $R$-EBUS, radial probe endobronchial ultrasound. 
TABLE 3. Accuracy of preoperative small biopsy specimens for predicting the presence or absence of high-grade adenocarcinomas in surgical specimens

\begin{tabular}{lcccc}
\hline \multicolumn{1}{c}{ Type } & Sensitivity, \% (95\% CI) & Specificity, \% (95\% CI) & PPV, \% (95\% CI) & NPV, \% (95\% CI) \\
\hline Micropapillary & $7.8(2.2-18.9)$ & $97.4(90.9-99.7)$ & $66.7(22.3-95.7)$ & $61.5(52.2-70.1)$ \\
Solid & $14.6(5.6-29.2)$ & $97.7(91.9-99.7)$ & $75.0(34.9-96.8)$ & $70.8(61.8-78.8)$ \\
High-grade subtypes & $16.5(9.1-26.5)$ & $98.0(89.2-99.9)$ & $92.9(66.1-99.8)$ & $42.1(32.9-51.7)$ \\
\hline
\end{tabular}

$C I$, Confidence interval; $P P V$, positive predictive value; $N P V$, negative predictive value.

amount of high-grade adenocarcinoma remains controversial, we demonstrated that the percentage of high-grade adenocarcinoma increased with increasing disease progression. In our cohort, the optimal cutoff value was $40 \%$, as estimated by the log-rank test.

Identifying high-grade adenocarcinoma in patients with early-stage lung adenocarcinoma at the preoperative stage is crucial. Only a limited number of studies have compared the efficacy of adenocarcinoma subtyping in paired small biopsy versus surgical resection. ${ }^{15,16,19,26}$ Among these, Matsuzawa et al ${ }^{19}$ reported a $66 \%$ concordance of predominant subtypes in 326 resected specimens and matched biopsy specimens; however, they did not analyze the concordance of the presence or absence of high-grade adenocarcinoma. In our study, we evaluated 128 patients to compare preoperative adenocarcinoma subtyping based on CTNB or R-EBUS biopsy samples of primary lung cancers with subtyping based on permanent resected specimens. The overall agreement was $58.6 \%$ for the predominant pattern, similar to that reported by Matsuzawa et $\mathrm{al}^{19}$; however, preoperative subtyping was accurate in only $30 \%$ of samples ( 3 of 10 ) with a predominance of solid patterns. Furthermore, none of the 5 micropapillary predominant cases was detected by CTNB or R-EBUS (sensitivity, 0) (Table 2). Our findings are slightly inferior compared with those reported previously by Yeh et $\mathrm{al}^{16}$ and Bittar et al, ${ }^{15}$ which showed an unsatisfactory predictive value of small frozen sections for adenocarcinoma subtyping.

Regarding the prediction of the presence or absence of high-grade adenocarcinoma, CTNB and R-EBUS preoperative biopsy specimens exhibited high specificity (micropapillary, 97.4\%; solid, 97.7\%), but very low sensitivity (7.8\% and $14.6 \%$, respectively) (Table 3), similar to values reported by Yeh et al ${ }^{16}$ and Bittar et al. ${ }^{15}$ When the micropapillary and solid subtypes are grouped together, CTNB and R-EBUS also can provide information on the presence of high-grade adenocarcinoma with high specificity but very low sensitivity. These findings illustrate that the high specificity is the result of a relatively lower detection rate of micropapillary and solid patterns $(7.8 \%$ and $14.6 \%$, respectively), rather than the exact preoperative detection by CTNB or R-EBUS.

Furthermore, the higher percentage of high-grade adenocarcinoma was associated with the higher rate of detection by CTNB/R-EBUS (Figure 3). However, even in the group of $\geq 40 \%$ micropapillary/solid components with a significant impact on DFS, only $24 \%$ could be detected by preoperative biopsy sections. This is because CTNB cannot always traverse the longest diameter of every tumor owing

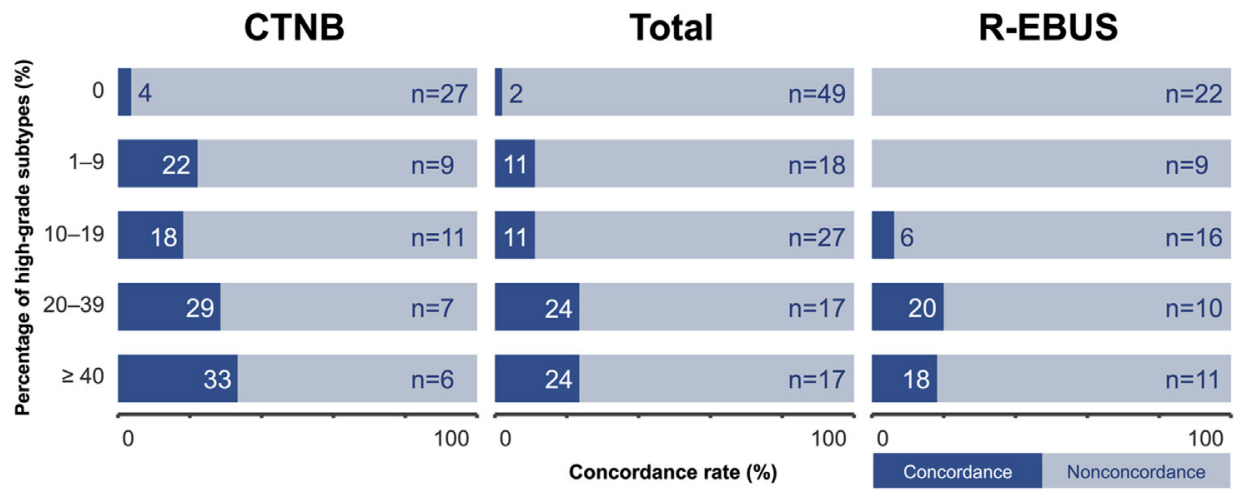

FIGURE 3. Percentage of detecting the presence or absence of micropapillary/solid component by preoperative biopsy sections. The detection rate by CTNB/R-EBUS increased with the increase in the percentage of the micropapillary/solid component. Of the 18 adenocarcinoma specimens with $1 \%$ to $9 \%$ micropapillary/solid component, $11 \%$ could be detected; of the 27 specimens with $10 \%$ to $19 \%$ micropapillary/solid component, $11 \%$ could be detected; of the 17 specimens with $20 \%$ to $39 \%$ micropapillary/solid component, $24 \%$ could be detected; of the 17 specimens with $\geq 40 \%$ micropapillary/ solid component, $24 \%$ could be detected (middle). For subgroup observation, CTNB was better than R-EBUS; however, even in the $\geq 40 \%$ micropapillary/ solid group, only $33 \%$ were detected by CTNB (left and right). CTNB, Computed tomography-guided 18-gauge core needle biopsy; $R$-EBUS, radial probe endobronchial ultrasound. 


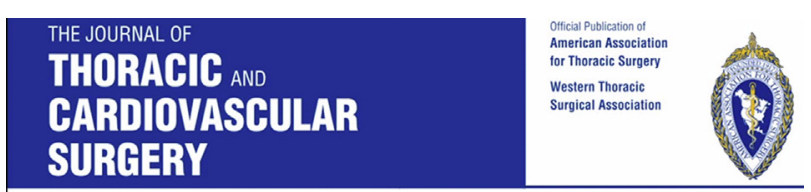

\section{Accuracy of lung adenocarcinoma subtyping using preoperative biopsy specimens}

\section{by KY Huang, et al.}

VIDEO 1. The predominant architectural subtype of lung adenocarcinoma is correlated with clinical prognosis; however, the accuracy of subtyping using preoperative biopsy specimens has not yet been completely validated. This video summarizes the rationale, study design, and main results of our research. Video available at: http://www.jtcvsonline.org/article/ S0022-5223(17)30421-X/addons.

to the tumor location in relation to the direction of needle approach and other surrounding structures. Even if CTNB could traverse the longest diameter of every tumor, the total volume of tissue retrieved in the core needle is fixed, and thus the extent of representative sampling in a given tumor volume is limited. Moreover, it is not possible to extensively burrow into and through the entire tumor using R-EBUS biopsy forceps.

The 2015 WHO adenocarcinoma histopathology classification system indicates a favorable correlation with CT findings. The GGOs correspond to the lepidic-like preinvasive components, and a part-solid or solid radiographic feature indicates invasive pathological components. ${ }^{27} \mathrm{Cha}$ et $\mathrm{al}^{8}$ reported that a solid mass on CT, tumor size of $\geq 2.5 \mathrm{~cm}$, maximal standardized uptake value of $\geq 7$ on PET scan, and tumor higher than stage I were independent predictors of the presence of high-grade adenocarcinoma components. In our study, stage (IA vs IB-IV) and tumor size had no influence on the concordance of identifying high-grade adenocarcinoma compared with paired preoperative biopsies and operative histologies. Radiologic parameters based on TDR were only slightly useful for predicting concordance, because CTNB/R-EBUS had higher specificity for detecting high-grade adenocarcinomas and a higher TDR reflected a lower amount of high-grade adenocarcinoma. In other words, the results would have been accurate when preoperative biopsy revealed the absence of micropapillary/solid components in high-TDR patients. However, surgeons cannot rely on the R-EBUS evaluation report indicating the absence of micropapillary/solid components and perform a sublobar resection when the CT scan shows a solid lung mass with lower TDR.

Various factors, including intratumoral histological heterogeneity, biopsy size, interpretation error, and degree of
TABLE 4. CT-detected factors influencing the accuracy of identifying high-grade adenocarcinomas via preoperative biopsy sections in $\mathbf{1 2 6}$ patients*

\begin{tabular}{lccc}
\hline \multicolumn{1}{c}{ Variable } & $\begin{array}{c}\text { Concordance } \\
(\mathbf{n = 6 1})\end{array}$ & $\begin{array}{c}\text { Nonconcordance } \\
(\mathbf{n = 6 5})\end{array}$ & $\begin{array}{c}\boldsymbol{P} \\
\text { value }\end{array}$ \\
\hline $\begin{array}{l}\text { Tumor size }(\mathrm{mm}) \text {, median } \\
\text { (IQR) }\end{array}$ & $27.5(21.1-35.0)$ & $29.9(22.0-37.3)$ & .26 \\
$\begin{array}{l}\text { Consolidation size (mm), } \\
\text { median (IQR) }\end{array}$ & $26.5(20.0-32.7)$ & $29.6(21.1-36.9)$ & .11 \\
TDR, median (IQR) & $0.37(0.22-0.54)$ & $0.26(0.17-0.38)$ & .02 \\
Spiculation, n (\%) & & & \\
$\quad$ Spiculated & $45(35.7)$ & $47(37.3)$ & .99 \\
$\quad$ Nonspiculated & $16(12.7)$ & $18(14.3)$ & \\
Lesion border, n (\%) & & & \\
$\quad$ Lobulated & $46(36.5)$ & $58(46.0)$ & .07 \\
$\quad$ Nonlobulated & $15(11.9)$ & $7(5.6)$ & \\
Margin, n (\%) & & & \\
Well-defined & $13(10.3)$ & $17(13.5)$ & .67 \\
Ill-defined & $48(38.1)$ & $48(38.1)$ & \\
Tumor solidity, n (\%) & & & \\
Solid & $45(35.7)$ & $57(45.2)$ & .09 \\
Partly solid & $14(11.1)$ & $8(6.3)$ & \\
Not solid (GGO) & $2(1.6)$ & $0(0.0)$ & \\
Stage, n (\%) & & & \\
IA & $20(15.9)$ & $16(12.7)$ & .41 \\
IB-IV & $41(32.5)$ & $49(38.9)$ & \\
\hline
\end{tabular}

$\overline{I Q R}$, Interquartile range; $T D R$, tumor shadow disappearance rate; $G G O$, ground glass opacity. *Two CT images were missing.

adenocarcinoma differentiation, can contribute to adenocarcinoma mistyping. ${ }^{15,16,18,19}$ We observed a more consistent agreement and higher sensitivity for the detection of highgrade adenocarcinomas with CTNB sections than with R-EBUS sections ( $24.2 \%$ vs $10.9 \%)$, although direct comparisons of these 2 biopsy methods were obscured by the uncontrolled biases. Regarding preoperative and intraoperative biopsy sample size, the frozen sections were larger than the CTNB sections, which in turn were larger than the R-EBUS sections. Compared with the results of previous studies, ${ }^{15,16}$ the overall agreement between preoperative frozen sections and permanent sections was better in the present study. Matsuzawa et $\mathrm{al}^{19}$ also found a significantly higher concordance rate in preoperative biopsy sections with larger tumor areas $\left(\geq 0.7 \mathrm{~mm}^{2}\right)$ compared with those with smaller tumor areas $\left(<0.7 \mathrm{~mm}^{2}\right)$. This finding confirmed previous observations that sampling error, rather than interpretation error by the pathologist, was the most likely reason for inaccurate subtyping. ${ }^{15,16}$

Our study had some limitations that should be addressed. First, there may be interexaminer variability among pathologists in lung adenocarcinoma subtyping. ${ }^{15,16}$ Because this was not the focus of the study, we did not evaluate this possibility further. Second, because this was a retrospective study, the patient population was relatively complex, with different tumor heterogeneity and 
preoperative diagnostic methods (CTNB or R-EBUS). Therefore, from our results, we could not identify the group of patients that could be accurately subtyped. Third, we did not analyze the correlation of high-grade adenocarcinoma and overall survival, because overall survival is influenced by various factors, including age, performance status, and various treatments (eg, local radiotherapy, target therapy, chemotherapy, immunotherapy), and our goal was to determine the accuracy between preoperative biopsy sections and permanent sections.

\section{CONCLUSIONS}

The results of this study indicate that the primary pattern of, or even the presence or absence of, high-grade adenocarcinoma cannot be predicted reliably based on CTNB or R-EBUS sections. Surgeons should not rely solely on preoperative CTNB or R-EBUS findings when making decisions regarding the choice of limited resection or lobectomy.

\section{Conflict of Interest Statement}

Authors have nothing to disclose with regard to commercial support.

\section{References}

1. Siegel RL, Miller KD, Jemal A. Cancer statistics, 2015. CA Cancer J Clin. 2015; 65:5-29.

2. Travis WD, Brambilla E, Nicholson AG, Yatabe Y, Austin JH, Beasley MB, et al. The 2015 World Health Organization classification of lung tumors: impact of genetic, clinical and radiologic advances since the 2004 classification. J Thorac Oncol. 2015;10:1243-60.

3. Travis WD, Brambilla E, Noguchi M, Nicholson AG, Geisinger KR, Yatabe Y, et al. International Association for the Study of Lung Cancer/American Thoracic Society/European Respiratory Society: international multidisciplinary classification of lung adenocarcinoma. J Thorac Oncol. 2011;6:244-85.

4. Nakamura H, Saji H, Shinmyo T, Tagaya R, Kurimoto N, Koizumi H, et al. Close association of IASLC/ATS/ERS lung adenocarcinoma subtypes with glucoseuptake in positron emission tomography. Lung Cancer. 2015;87:28-33.

5. Tsao MS, Marguet S, Le Teuff G, Lantuejoul S, Shepherd FA, Seymour L, et al. Subtype classification of lung adenocarcinoma predicts benefit from adjuvant chemotherapy in patients undergoing complete resection. J Clin Oncol. 2015; 33:3439-46.

6. Ujiie H, Kadota K, Chaft JE, Buitrago D, Sima CS, Lee MC, et al. Solid predominant histologic subtype in resected stage I lung adenocarcinoma is an independent predictor of early, extrathoracic, multisite recurrence and of poor postrecurrence survival. J Clin Oncol. 2015;33:2877-84.

7. Lee G, Lee HY, Jeong JY, Han J, Cha MJ, Lee KS, et al. Clinical impact of minimal micropapillary pattern in invasive lung adenocarcinoma: prognostic significance and survival outcomes. Am J Surg Pathol. 2015;39:660-6.

8. Cha MJ, Lee HY, Lee KS, Jeong JY, Han J, Shim YM, et al. Micropapillary and solid subtypes of invasive lung adenocarcinoma: clinical predictors of histopathology and outcome. J Thorac Cardiovasc Surg. 2014;147:921-8.e2.

9. Zhang Y, Sun Y, Xiang J, Zhang Y, Hu H, Chen H. A clinicopathologic prediction model for postoperative recurrence in stage Ia non-small cell lung cancer. $J$ Thorac Cardiovasc Surg. 2014;148:1193-9.

10. Yeh YC, Kadota K, Nitadori J, Sima CS, Rizk NP, Jones DR, et al. International Association for the Study of Lung Cancer/American Thoracic Society/European Respiratory Society classification predicts occult lymph node metastasis in clinically mediastinal node-negative lung adenocarcinoma. Eur J Cardiothorac Surg. 2016;49:e9-15.

11. Hung JJ, Yeh YC, Jeng WJ, Wu YC, Chou TY, Hsu WH. Factors predicting occult lymph node metastasis in completely resected lung adenocarcinoma of $3 \mathrm{~cm}$ or smaller. Eur J Cardiothorac Surg. 2016;50:329-36.
12. Ramnath N, Dilling TJ, Harris LJ, Kim AW, Michaud GC, Balekian AA, et al. Treatment of stage III non-small cell lung cancer: diagnosis and management of lung cancer, 3rd ed: American College of Chest Physicians evidence-based clinical practice guidelines. Chest. 2013;143(5 Suppl): e314S-40S.

13. Oki M, Saka H, Ando M, Kitagawa C, Kogure Y, Seki Y. Endoscopic ultrasound-guided fine-needle aspiration and endobronchial ultrasoundguided transbronchial needle aspiration: are two better than one in mediastinal staging of non-small cell lung cancer? J Thorac Cardiovasc Surg. 2014;148:1169-77.

14. Sesti J, Donington JS. Sublobar resection: ongoing controversy for treatment for stage I non-small cell lung cancer. Thorac Surg Clin. 2016;26:251-9.

15. Trejo Bittar HE, Incharoen $P$, Althouse AD, Dacic S. Accuracy of the IASLC ATS/ERS histological subtyping of stage I lung adenocarcinoma on intraoperative frozen sections. Mod Pathol. 2015;28:1058-63.

16. Yeh YC, Nitadori J, Kadota K, Yoshizawa A, Rekhtman N, Moreira AL, et al. Using frozen section to identify histological patterns in stage I lung adenocarcinoma of $\leq 3 \mathrm{~cm}$ : accuracy and interobserver agreement. Histopathology. 2015; 66:922-38.

17. Sigel CS, Moreira AL, Travis WD, Zakowski MF, Thornton RH, Riely GJ, et al. Subtyping of non-small cell lung carcinoma: a comparison of small biopsy and cytology specimens. J Thorac Oncol. 2011;6: 1849-56.

18. Yamagishi T, Shimizu K, Ochi N, Yamane H, Irei I, Sadahira Y, et al. Histological comparison between preoperative and surgical specimens of non-small cell lung cancer for distinguishing between "squamous" and "non-squamous" cell carcinoma. Diagn Pathol. 2014;9:103.

19. Matsuzawa R, Kirita K, Kuwata T, Umemura S, Matsumoto S, Fujii S, et al Factors influencing the concordance of histological subtype diagnosis from biopsy and resected specimens of lung adenocarcinoma. Lung Cancer 2016;94:1-6.

20. Takahashi M, Shigematsu Y, Ohta M, Tokumasu H, Matsukura T, Hirai T. Tumor invasiveness as defined by the newly proposed IASLC/ATS/ERS classification has prognostic significance for pathologic stage IA lung adenocarcinoma and can be predicted by radiologic parameters. J Thorac Cardiovasc Surg. 2014; 147:54-9.

21. Landreneau RJ, Normolle DP, Christie NA, Awais O, Wizorek JJ, Abbas G, et al. Recurrence and survival outcomes after anatomic segmentectomy versus lobectomy for clinical stage I non-small-cell lung cancer: a propensity-matched analysis. J Clin Oncol. 2014;32:2449-55.

22. Hung JJ, Yeh YC, Jeng WJ, Wu KJ, Huang BS, Wu YC, et al. Predictive value of the International Association for the Study of Lung Cancer/American Thoracic Society/European Respiratory Society classification of lung adenocarcinoma in tumor recurrence and patient survival. J Clin Oncol. 2014:32:2357-64.

23. Nitadori J, Bograd AJ, Kadota K, Sima CS, Rizk NP, Morales EA, et al Impact of micropapillary histologic subtype in selecting limited resection vs lobectomy for lung adenocarcinoma of $2 \mathrm{~cm}$ or smaller. J Natl Cancer Inst. 2013;105:1212-20.

24. Tsutani Y, Miyata Y, Mimae T, Kushitani K, Takeshima Y, Yoshimura M, et al The prognostic role of pathologic invasive component size, excluding lepidic growth, in stage I lung adenocarcinoma. J Thorac Cardiovasc Surg. 2013;146: 580-5.

25. Zhao ZR, To KF, Mok TS, Ng CS. Is there significance in identification of nonpredominant micropapillary or solid components in early-stage lung adenocarcinoma? Interact Cardiovasc Thorac Surg. 2017;24:121-5.

26. Ferretti GR, Busser B, de Fraipont F, Reymond E, McLeer-Florin A, MescamMancini L, et al. Adequacy of CT-guided biopsies with histomolecular subtyping of pulmonary adenocarcinomas: influence of ATS/ERS/IASLC guidelines. Lung Cancer. 2013;82:69-75.

27. Cohen JG, Reymond E, Jankowski A, Brambilla E, Arbib F, Lantuejoul S, et al. Lung adenocarcinomas: correlation of computed tomography and pathology findings. Diagn Interv Imaging. 2016;97:955-63.

Key Words: computed tomography-guided 18-gauge core needle biopsy (CTNB), high-grade adenocarcinoma, lung adenocarcinoma, radial probe endobronchial ultrasound (R-EBUS), subtype 


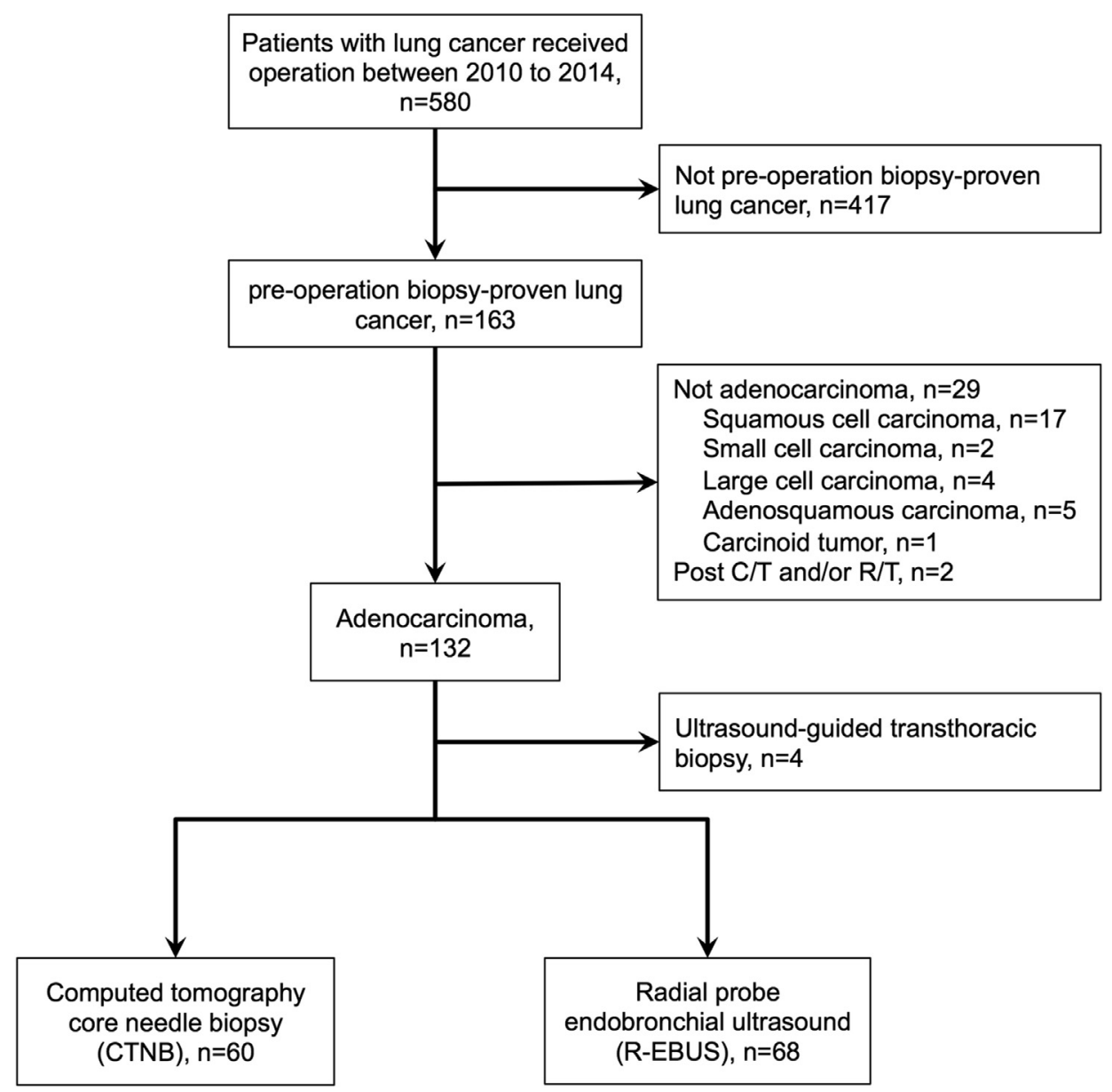

FIGURE E1. Flowchart of enrolled subjects. $C / T$, Chemotherapy; $R / T$, radiotherapy; $C T N B$, computed tomography-guided 18-gauge core needle biopsy; $R$-EBUS, radial probe endobronchial ultrasound.

TABLE E1. Log-rank test to determine the cutoff threshold percentage of high-grade adenocarcinomas

\begin{tabular}{|c|c|c|c|c|c|c|c|c|c|}
\hline Stage & $\geq 10 \%$ & $\geq \mathbf{2 0} \%$ & $\geq \mathbf{3 0} \%$ & $\geq \mathbf{4 0} \%$ & $\geq \mathbf{5 0} \%$ & $\geq \mathbf{6 0} \%$ & $\geq \mathbf{7 0} \%$ & $\geq \mathbf{8 0} \%$ & $\geq \mathbf{9 0} \%$ \\
\hline I & 0.0677 & 0.1544 & 0.0371 & 0.0003 & 0.0003 & 0.3539 & 0.3248 & 0.3248 & NA \\
\hline II & 0.7582 & 0.9858 & 0.7097 & 0.9299 & 0.3818 & 0.5188 & NA & NA & NA \\
\hline
\end{tabular}

NA, Not available. 